\title{
Post Neoadjuvant Therapy Sample
}

National Cancer Institute

\section{Source}

National Cancer Institute. Post Neoadjuvant Therapy Sample. NCI Thesaurus. Code C156444.

A biospecimen derived from a subject after treatment with a neoadjuvant therapeutic. 